\title{
Rule-based versus probabilistic selection for active surveillance using three definitions of insignificant prostate cancer
}

\author{
Lionne D. F. Venderbos ${ }^{1,2} \cdot$ Monique J. Roobol $^{1} \cdot$ Chris H. Bangma $^{1}$. \\ Roderick C. N. van den Bergh ${ }^{1}$ - Leonard P. Bokhorst ${ }^{1} \cdot$ Daan Nieboer $^{2}$. \\ Rebecka Godtman ${ }^{3} \cdot$ Jonas Hugosson $^{3} \cdot$ Theodorus van der Kwast $^{4}$. \\ Ewout W. Steyerberg 2
}

Received: 8 May 2015 / Accepted: 22 June 2015 / Published online: 10 July 2015

(C) The Author(s) 2015. This article is published with open access at Springerlink.com

\begin{abstract}
Purpose To study whether probabilistic selection by the use of a nomogram could improve patient selection for active surveillance (AS) compared to the various sets of rule-based AS inclusion criteria currently used.

Methods We studied Dutch and Swedish patients participating in the European Randomized study of Screening for Prostate Cancer (ERSPC). We explored which men who were initially diagnosed with cT1-2, Gleason 6 (Gleason pattern $\leq 3+3$ ) had histopathological indolent $\mathrm{PCa}$ at RP [defined as pT2, Gleason pattern $\leq 3$ and tumour volume (TV) $\leq 0.5$ or TV $\leq 1.3 \mathrm{ml}$, and TV no part of criteria (NoTV)]. Rule-based selection was according to the Prostate cancer Research International: Active Surveillance (PRIAS), Klotz, and Johns Hopkins criteria. An existing nomogram to define probability-based selection for AS was refitted for the TV1.3 and NoTV indolent PCa definitions. Results 619 of 864 men undergoing RP had cT1-2, Gleason 6 disease at diagnosis and were analysed. Median
\end{abstract}

Electronic supplementary material The online version of this article (doi:10.1007/s00345-015-1628-y) contains supplementary material, which is available to authorized users.

Lionne D. F. Venderbos

l.venderbos@erasmusmc.nl

1 Department of Urology, Erasmus University Medical Center, Room Na1710, P.O. Box 2040, 3000 CA Rotterdam, The Netherlands

2 Department of Public Health, Erasmus University Medical Center, Rotterdam, The Netherlands

3 Department of Urology, Sahlgrenska Academy at Göteborg University, Göteborg, Sweden

4 Department of Pathology, Toronto General Hospital, University Health Network, Toronto, Canada follow-up was 8.9 years. 229 (37\%), $356(58 \%)$, and $410(66 \%)$ fulfilled the TV0.5, TV1.3, and NoTV indolent PCa criteria at RP. Discriminating between indolent and significant disease according to area under the curve (AUC) was: TV0.5: 0.658 (PRIAS), 0.523 (Klotz), 0.642 (Hopkins), 0.685 (nomogram). TV1.3: 0.630 (PRIAS), 0.550 (Klotz), 0.615 (Hopkins), 0.646 (nomogram). NoTV: 0.603 (PRIAS), 0.530 (Klotz), 0.589 (Hopkins), 0.608 (nomogram).

Conclusions The performance of a nomogram, the Johns Hopkins, and PRIAS rule-based criteria are comparable. Because the nomogram allows individual trade-offs, it could be a good alternative to rigid rule-based criteria.

Keywords Prostatic neoplasm · Active surveillance · Risk stratification - Selection for active surveillance . Inclusion criteria active surveillance $\cdot$ Nomogram

\section{Introduction}

Early detection of prostate cancer $(\mathrm{PCa})$ has led to increased prevalence of finding indolent tumours, i.e. tumours that are unlikely to become symptomatic during life. The ability to predict indolent PCa is needed to avoid overtreatment [1]. Active surveillance (AS) has emerged as a feasible strategy to decrease the overtreatment of low-risk $\mathrm{PCa}$. With AS, men with low-risk PCa are strictly monitored over time, and if risk reclassification or disease progression occurs, they can opt for curative therapy. Hence, the aim of AS is to safely delay or completely avoid side effects of active therapy [2]. There are 16 unique worldwide AS cohorts which all have their highly variable own protocols [3]. So far, published results on AS study cohorts worldwide show encouraging results on biochemical 
recurrence (BCR) rates and disease-specific mortality [4]. Long-term effects are yet unknown. Research on how to improve the existing AS protocols is, however, needed as misclassification at diagnosis, and subsequent reclassification after one-year repeat biopsy is not uncommon [5]. For example, $28 \%$ of men within the Prostate cancer Research International: Active Surveillance (PRIAS) study were reclassified after one or more repeat biopsies [6].

Currently, all existing AS cohorts apply relatively simple combinations of inclusion criteria for patient selection ("rule-based selection"). More refined risk stratification through a nomogram may be preferable, especially in the light of individualised medicine and shared decision-making ("probability-based selection") [7]. We aimed to assess the performance of inclusion criteria as used in several prospective AS protocols in identifying indolent cancer at radical prostatectomy (RP) and follow-up outcomes of men who received immediate RP but were also suitable for AS. For comparison, we used a previously developed and externally validated nomogram that predicts indolent disease $[8$, 9]. We hypothesise that the use of probabilistic selection by the use of a nomogram that incorporates multiple patient characteristics may be better for selection.

\section{Materials and methods}

\section{Patients}

Men included in this study were participants in the screening arm of the European Randomized study of Screening for Prostate Cancer (ERSPC). Data cohorts of the Swedish and Dutch sections of ERSPC were combined. All men were diagnosed with screen-detected $\mathrm{PCa}$ and underwent RP as primary treatment. Details on both Dutch and Swedish screening protocols were previously published $[10,11]$.

\section{Methods}

Men with T3-4, Gleason $\geq 7$ PCa at diagnostic biopsy or an unknown tumour volume were excluded from this analysis, as well as men with positive lymph nodes or distant metastases at the time of diagnosis or at the time of surgery. A multiple imputation model was used to fill in missing data. We used the first imputation of a multiple imputation procedure with the impute function in SPSS software (IBM Corp. Released 2012. IBM SPSS Statistics for Windows, version 21.0. Armonk, NY: IBM Corp). A total of 936 confounder values were missing, comprising $13.5 \%$ of all values. Filling in these values through imputation allowed us to include the $382(44 \%)$ patients with any missing value in the analysis. All tumour characteristics were used for the multiple imputation.
We first assessed the frequency of indolent PCa at RP according to the classic definition of pT2, tumour volume $<0.5 \mathrm{ml}$ (TV0.5), and pathological Gleason pattern $\leq 3$ [12]. Men not fulfilling these criteria for indolent $\mathrm{PCa}$ (TV $>0.5 \mathrm{ml}$ and/or pathological Gleason pattern $>3$ ) were categorised as having significant PCa.

Second, we selected men from our study cohort with low-risk PCa at diagnosis defined according to the PRIAS (T1c-T2, PSA $\leq 10 \mathrm{ng} / \mathrm{ml}$; PSA density $<0.20 \mathrm{ng} / \mathrm{ml} /$ cc, Gleason $\leq 3+3, \leq 2$ positive cores), Klotz (T1b-T2b; PSA $\leq 10 \mathrm{ng} / \mathrm{ml}$; Gleason $\leq 6$ ), and Johns Hopkins criteria (T1c, PSA density $<0.15 \mathrm{ng} / \mathrm{ml} / \mathrm{cc}$, Gleason $\leq 6, \leq 2$ positive cores, $\leq 50 \%$ single core involvement). The frequencies of indolent PCa at RP in these groups were studied.

Third, we explored the use of a nomogram to estimate risk for indolent PCa at RP [13]. We assessed the effect of applying various eligibility criteria for the nomogram (T1cT2a, PSA $\leq 20 \mathrm{ng} / \mathrm{ml}$; Gleason $\leq 3+3, \leq 50 \%$ positive cores, $20 \mathrm{~mm}$ PCa, $40 \mathrm{~mm}$ benign tissue in all cores) and of different thresholds in the predicted chance of harbouring indolent $\mathrm{PCa}$ (referred to as Pind) on the number of men remaining suitable for AS at diagnosis.

The classic definition of a pathologic indolent $\mathrm{PCa}$ (pT2, TV0.5, and Gleason pattern $\leq 3$ ) might be too restrictive and not reflecting biology well [14]. Therefore, we repeated steps one to three with two updated and more recent definitions of indolent PCa: (1) pT2, tumour volume $<1.3 \mathrm{ml}$ (TV1.3) and Gleason pattern $\leq 3+3$ [14-16]; (2) pT2, Gleason pattern $\leq 3+3$ and tumour volume no part of definition (NoTV) [15]. For step three, the nomogram was refitted twice using the original data [13], to account for the adjusted definitions of an indolent PCa.

Having the availability of follow-up data, we were able to calculate BCR after RP. The criteria proposed by Freedland et al. [17] were used to define BCR, i.e. one PSA value after RP $>0.2 \mathrm{ng} / \mathrm{ml}$. The different sets of rule-based selection criteria and Pind cut-off points were compared using the Kaplan-Meier method and the log-rank test.

We finally applied decision curve analysis (DCA) [18] to evaluate the potential clinical usefulness of rule-based selection and probability-based selection models. We estimated a net benefit (NB) for the four models by summing the benefits (true-positive indolent PCa) and subtracting the harms (falsepositive indolent $\mathrm{PCa}$ ). The harms were weighted by a factor related to the relative harm of being unjustly included on AS versus being directly curatively treated while suitable for AS. This weighting was derived from the threshold probability at which a patient would opt for AS. This threshold varies between men and urologists. Clinical practice currently uses a threshold probability of 50-70\% [19]. The interpretation of a decision curve is rather straightforward; the model with the highest NB at a particular threshold should be chosen over alternative models. 
$P$ values (two-sided) $<0.05$ were considered statistically significant. For statistical analysis, we used the Statistical Package for the Social Sciences (SPSS) version 21 (IBM Corp. Released 2012. IBM SPSS Statistics for Windows, version 21.0. Armonk, NY: IBM Corp) and R version 2.15.2 (R Foundation for Statistical Computing, Vienna, Austria).

\section{Results}

Our study cohort consisted of 864 men of whom 619 had cT1-2, Gleason 6 disease at diagnosis and were therefore eligible for analyses. Median follow-up time after diagnosis was 8.9 years. Table 1 presents the study cohort characteristics at diagnosis and outcomes after RP. With
Table 1 Study cohort characteristics at diagnosis and outcomes after radical prostatectomy $(n=619)$

\section{At diagnosis \\ ERSPC study centre:}

The Netherlands $(n, \%) \quad 336 \quad 54$

Sweden $(n, \%)$

$283 \quad 46$

Follow-up (years) (median, 25-75p)

$8.9 \quad 5.9-10.9$

Age (years) (median, 25-75p)

$62.9 \quad 60.1-66.2$

Clinical disease stage $(n, \%)$ :

T1c

$395 \quad 64$

T2a

$\mathrm{T} 2 \mathrm{~b}$

$\mathrm{T} 2 \mathrm{c}$

PSA (ng/ml) (median, 25-75p)

Prostate volume (cc; median, 25-75p)

$157 \quad 25$

$37 \quad 6$

$30 \quad 5$

$4.5 \quad 3.5-6.4$

$35.1 \quad 28.3-45.1$

PSA density (ng/ml/cc; median, 25-75p)

Total number of cores (median, 25-75p)

Number of positive cores (median, 25-75p)

$0.13 \quad 0.09-0.18$

$6 \quad 6-6$

$2 \quad 1-3$

Total benign tissue (mm; median, 25-75p)

$67.6 \quad 56.0-78.5$

Total PCa tissue (mm; median, 25-75p)

$4.0 \quad 2.1-8.1$

Percentage cancer per positive core (median, 25-75p)

$30.5 \quad 14.7-64.3$

Gleason sum:

$\leq 6$

$619 \quad 100$

Prediction indolent cancer (median, $25-75 p)(n=455$ suitable for nomogram)

$60 \% \quad 40-78 \%$

At radical prostatectomy

Tumour volume $(n, \%)$

$\leq 0.5 \mathrm{cc}$

$>0.5 \mathrm{cc}$

284

335

497

$>1.3 \mathrm{cc}$

Gleason sum $(n, \%)$

$\leq 6$ (no pattern 4$)$

$\geq 7$

Indolent cancer (tumour volume $\leq 0.5 \mathrm{cc}, \mathrm{T} 2$, Gleason $\leq 3+3$ disease)

Yes $(n, \%)$

229

No $(n, \%)$

Indolent cancer (tumour volume $\leq 1.3 \mathrm{cc}, \mathrm{T} 2$, Gleason $\leq 3+3$ disease)

Yes $(n, \%)$

$356 \quad 58$

No $(n, \%)$

263

42

Indolent cancer ( 2 , Gleason $\leq 3+3$ disease, no tumour volume cut-off)

Yes $(n, \%)$

$410 \quad 66$

No $(n, \%)$

209

34

25-75p 25-75 percentile 
TV0.5 cut-off, a total of 229 (37\%) tumours at RP could be defined as indolent versus $390(63 \%)$ as significant. When applying the TV1.3 and NoTV indolent PCa definitions, the number of indolent PCa increases to 356 (58) and 410 (66 \%), respectively. Pind could be calculated for 455 (74\%) men meeting the nomogram inclusion criteria.

Table 2 presents the sensitivity, specificity, positive predictive value (PPV), and negative predictive value (NPV) for all three indolent PCa definitions (TV0.5, TV1.3, NoTV) at RP of the rule-based selection and nomogrambased selection approaches. The table also contains the effect of applying different thresholds of the nomogram calculated risk of harbouring indolent PCa, i.e. Pind.

The area under the curve (AUC) for the TV0.5 indolent definition was 0.658 for PRIAS, 0.523 for Klotz, 0.642 for Johns Hopkins, and 0.685 for the nomogram. For the TV1.3 indolent definition, the AUC for PRIAS was 0.630, for Klotz 0.550, for Johns Hopkins 0.615, and for the refitted nomogram 0.646. For the NoTV indolent definition, the AUC for PRIAS was 0.603, for Klotz 0.530, for Johns Hopkins 0.589 , and for the refitted nomogram 0.608.

Table 2 furthermore presents the number of men who experienced BCR after RP according to the three definitions of indolent disease in the different sets of rule-based criteria and the nomogram suitable cohort. A log-rank test showed that the number of men experiencing BCR do not differ statistically between the groups. However, the distribution of BCR over the indolent and significant group changes, with a rising percentage of BCR in the indolent group $(\mathrm{TV} 0.5=3.4 \%$, TV1.3 $=4.9 \%$, NoTV $=6.3 \%)$. We found that in ROC analysis (Appendix Fig. 1), the nomogram (TV0.5) had a slightly better sensitivity-to-specificity ratio than the PRIAS rules. The AUC for the nomogram (TV0.5) was 0.610 , for PRIAS 0.584, for Klotz 0.524, for Johns Hopkins 0.615, for the refitted TV1.3 nomogram 0.595 , and for the refitted NoTV nomogram 0.570.

In terms of clinical usefulness, we found that in DCA analysis (appendix Fig. 2a-c), no large differences in NB were seen for threshold probabilities 50-70\%, which are clinically most relevant.

\section{Discussion}

In our cohort of Dutch and Swedish screen-detected PCa patients who all underwent initial RP, $37 \%$ fulfilled the TV0.5 indolent PCa criteria at RP increasing to $58 \%$ for the TV1.3 indolent PCa criteria and $66 \%$ for the NoTV indolent $\mathrm{PCa}$ definition. More stringent rule-based AS inclusion criteria as well as stricter nomogram probability thresholds decrease the rate of misclassified tumours in a rather similar fashion, but both at the cost of a substantial number of patients no longer considered suitable for AS.
The nomogram based on TV0.5 had slightly better sensitivity and specificity with respect to BCR outcome than the PRIAS and Klotz criteria. If we juxtapose the TV0.5 nomogram to the Johns Hopkins criteria, the latter performed better but at the cost of including less patients and thereby curatively treating patients that might also would have been suitable for AS.

On the basis of a Kaplan-Meier analysis (curves not shown), we cannot conclude that the use of the TV0.5 nomogram is preferred over the use of rule-based selection or vice versa. However, for BCR the TV0.5 nomogram outperformed the PRIAS and Klotz criteria. The TV0.5 nomogram, however, performed slightly worse than the Johns Hopkins criteria. If we chose a slightly lower Pind and therewith allowing more men to be included on AS, sensitivity and specificity of the TV 0.5 nomogram are still acceptable. This flexibility in application is a property of using a nomogram for selection rather than a strict set of rules and desirable in the light of individualised medicine and shared decision-making.

Because the classic definition of a pathologically indolent PCa may be too restrictive [14], we also used two more updated definitions of an indolent PCa. When juxtaposing the models, the TV0.5 nomogram (AUC 0.685) was slightly better in discriminating indolent from significant PCa than the PRIAS (AUC 0.658), Johns Hopkins (AUC 0.642), and Klotz (AUC 0.523) criteria. This trend of the nomogram predicting slightly better is also seen for the refitted TV1.3 and NoTV nomograms.

Perfect patient selection for AS using either rule-based selection criteria or by applying a nomogram seems difficult at present. The AUCs illustrate that both approaches are currently suboptimal in differentiating indolent from non-indolent disease at RP in a group of men with already low-risk features at diagnosis. This is confirmed by the study of Wang et al. [20] whom in a group of 273 AS patients who underwent multiple biopsies and/or delayed RP found that nomograms designed to predict indolent tumours only have a modest ability to predict biopsy progression and any progression on either biopsy or surgery in men choosing an AS management strategy. Wang et al. furthermore concluded that in a subgroup of 58 men, none of the various nomograms were able to predict surgical progression at RP [20]. Since AS is incorporated into many guidelines (AUA, NCCN, EAU, etc.) as a viable management strategy for men with either very low-risk or low-risk $\mathrm{PCa}$, it is expected that more men will elect AS as their primary therapy. The optimisation of both rule-based selection and probability-based selection is therefore warranted.

Over the past few years, magnetic resonance imaging (MRI) is emerging as a tool which may be able to more accurately determine the risk of significant disease and progression of disease over time by improving sampling 


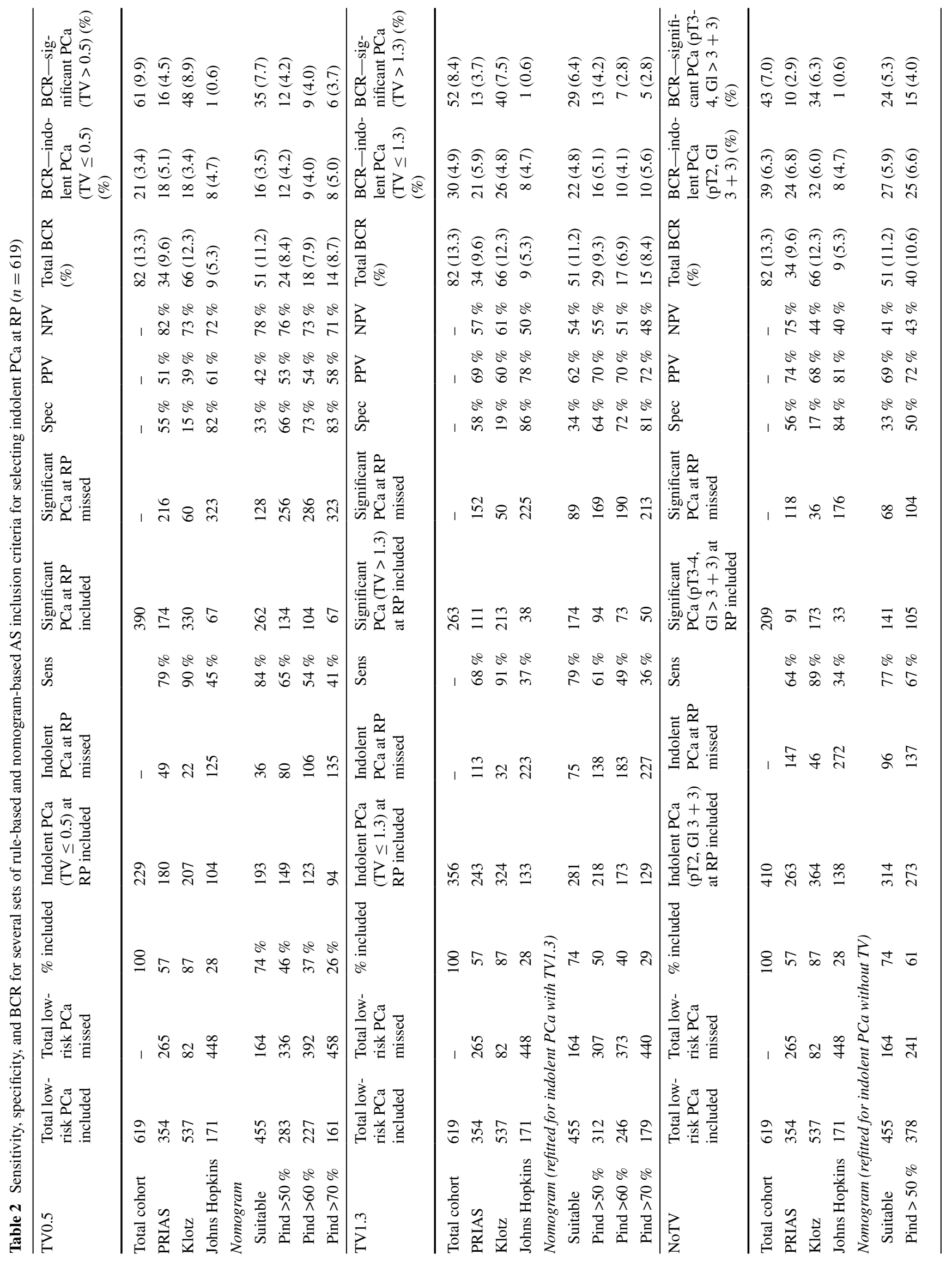


through target biopsies [21]. MRI may therefore also help better select AS candidates [22]. Several studies have shown the additional value of MRI in an AS protocol [21-23]. Stamatakis et al. [22] combined MRI-based factors into a nomogram which generates a probability for confirmed AS candidacy. They found that three MRI-based factors, i.e. number of lesions, lesion suspicion, and lesion density, were associated with confirmatory biopsy outcome and reclassification. A created nomogram which uses these factors has promising predictive accuracy, according to Stamatakis et al. [22]. It could be that adding such factors to the currently existing rule-based selection criteria or the nomogram could improve sensitivity and specificity and therewith AS patient selection.

A first limitation of our study lies in the fact that men in our cohort were diagnosed with sextant biopsies. Sextant biopsy does not reflect current clinical practice anymore; nowadays, current practice relies on 8-18 core biopsies. Studies that applied more extended biopsy schemes argue that with a sextant biopsy protocol, 10-30\% of cancers are missed [24]. Several studies reported that when 8-12 cores were taken, the PCa detection rate in a clinical setting might increase [24, 25]. We validated the previously developed nomogram in multiple other populations in which more extended biopsy schemes were used. Results of these validation studies showed that the nomogram predicted indolent $\mathrm{PCa}$ with good discrimination, indicating that it can be broadly applied in contemporary urological practice [26, 27]. In addition, we extracted correction factors for the adjustment of the nomogram with which contemporary extended biopsy schemes can be addressed [28]. Another limitation is that follow-up time of our study cohort is too short to assess mortality outcomes and relate these to baseline selection criteria. The lack of mortality outcomes was also the reason to choose BCR as an endpoint instead. Many men with BCR, however, will never develop metastasised disease or die from PCa [29]. Thirdly, patients underwent RP in different centres in either Sweden or the Netherlands. They were operated by different surgeons using different techniques for RP, which might influence outcomes. Finally, 247 cases included in this analysis were also used in the validation and construction of the nomogram. This may lead to an overestimated performance of the nomogram and Pind. The strength of this study lies in the fact that all men were diagnosed with PCa within ERSPC (Sweden and the Netherlands), resulting in standardised pathological examination of biopsy specimens and structured data follow-up [30].

In conclusion, in our cohort of Dutch and Swedish screen-detected PCa patients who all underwent initial RP, $37 \%$ had TV0.5 indolent PCa at RP increasing to $58 \%$ for the TV1.3 indolent PCa criteria and $66 \%$ for the NoTV indolent PCa definition. Performance of an 
ERSPC-based TV0.5 nomogram and rule-based selection by the Johns Hopkins and PRIAS criteria is comparable. Because the nomogram allows individual tradeoffs, it could be a good alternative to applying rigid rule-based criteria. Furthermore, a nomogram anticipates on the continuous improvement of risk assessment by newly emerging risk criteria, including imaging modalities.

Acknowledgments The international coordination of the European Randomized Study of Screening for Prostate Cancer (ERSPC) has been supported since the study's initiation in 1991 by grants from Europe Against Cancer and the fifth and sixth framework programme of the European Union, by many grants from agencies in the individual participating countries, and by unconditional grants from Beckman Coulter Hybritech Inc. Data referred to in this report are derived from ERSPC Rotterdam and Sweden. ERSPC Rotterdam has been supported by grants from the Dutch Cancer Society, the Netherlands Organisation for Health Research and Development, and the Abe Bonnema Foundation, as well as by many private donations. ERSPC Sweden has been supported by grants from Abbott Pharmaceuticals, Sweden; Af Jochnick's foundation; Catarina and Sven Hagstroms family foundation; Gunvor and Ivan Svensson's foundation; Johanniterorden, King Gustav V Jubilée Clinic Cancer Research Foundation; Sahlgrenska University Hospital, Schering Plough, Sweden; Swedish Cancer Society, Wallac Oy, Turku, Finland.

\section{Compliance with Ethical Standards}

Conflict of interest The authors have nothing to disclose.

Open Access This article is distributed under the terms of the Creative Commons Attribution 4.0 International License (http://creativecommons.org/licenses/by/4.0/), which permits unrestricted use, distribution, and reproduction in any medium, provided you give appropriate credit to the original author(s) and the source, provide a link to the Creative Commons license, and indicate if changes were made.

\section{References}

1. Bangma CH, Roobol MJ, Steyerberg EW (2009) Predictive models in diagnosing indolent cancer. Cancer 115(13 Suppl):3100-3106

2. van den Bergh RC, Roemeling S, Roobol MJ, Roobol W, Schroder FH, Bangma CH (2007) Prospective validation of active surveillance in prostate cancer: the PRIAS study. Eur Urol 52(6):1560-1563

3. Ip S, Dahabreh IJ, Chung M et al (2011) An evidence review of active surveillance in men with localized prostate cancer. Evid Rep Technol Assess (Full Rep). 204:1-341

4. Venderbos LD, Bokhorst LP, Bangma CH, Roobol MJ (2013) Active surveillance: oncologic outcome. Curr Opin Urol 23(3):268-272

5. Bul M, van den Bergh RC, Rannikko A et al (2012) Predictors of unfavourable repeat biopsy results in men participating in a prospective active surveillance program. Eur Urol 61(2):370-377 (Epub 2011/06/28)

6. Bul M, Zhu X, Valdagni R et al (2013) Active surveillance for low-risk prostate cancer worldwide: the PRIAS study. Eur Urol 63(4):597-603
7. Gandaglia G, Giannarini G, Suardi N, Montorsi F, Briganti A (2014) Will active surveillance for clinically localized prostate cancer survive in the era of individualized medicine? Eur Urol 66(2):186-187

8. Louie-Johnsun M, Neill M, Treurnicht K, Jarmulowicz M, Eden C (2009) Final outcomes of patients with low-risk prostate cancer suitable for active surveillance but treated surgically. BJU Int 104(10):1501-1504

9. Suardi N, Capitanio U, Chun FK et al (2008) Currently used criteria for active surveillance in men with low-risk prostate cancer: an analysis of pathologic features. Cancer 113(8):2068-2072

10. Roobol MJ, Kirkels WJ, Schroder FH (2003) Features and preliminary results of the Dutch centre of the ERSPC (Rotterdam, the Netherlands). BJU Int 92(Suppl 2):48-54

11. Hugosson J, Aus G, Bergdahl S et al (2003) Population-based screening for prostate cancer by measuring free and total serum prostate-specific antigen in Sweden. BJU Int 92(Suppl 2):39-43

12. Epstein JI, Walsh PC, Carmichael M, Brendler CB (1994) Pathologic and clinical findings to predict tumor extent of nonpalpable (stage t1 c) prostate cancer. JAMA 271(5):368-374

13. Steyerberg EW, Roobol MJ, Kattan MW, van der Kwast TH, de Koning HJ, Schroder FH (2007) Prediction of indolent prostate cancer: validation and updating of a prognostic nomogram. J Urol 177(1):107-112 (discussion 12)

14. Wolters T, Roobol MJ, van Leeuwen PJ et al (2011) A critical analysis of the tumor volume threshold for clinically insignificant prostate cancer using a data set of a randomized screening trial. J Urol 185(1):121-125

15. Van der Kwast TH, Roobol MJ (2013) Defining the threshold for significant versus insignificant prostate cancer. Nat Rev Urol. 10(8):473-482

16. Iremashvili V, Pelaez L, Manoharan M, Jorda M, Rosenberg DL, Soloway MS (2012) Pathologic prostate cancer characteristics in patients eligible for active surveillance: a head-to-head comparison of contemporary protocols. Eur Urol 62(3):462-468

17. Freedland SJ, Sutter ME, Dorey F, Aronson WJ (2003) Defining the ideal cutpoint for determining PSA recurrence after radical prostatectomy. Prostate-specific antigen. Urology. 61(2):365-369

18. Vickers AJ, Elkin EB (2006) Decision curve analysis: a novel method for evaluating prediction models. Med Decis Mak 26(6):565-574

19. van Vugt HA, Roobol MJ, van der Poel HG et al (2012) Selecting men diagnosed with prostate cancer for active surveillance using a risk calculator: a prospective impact study. BJU Int 110(2): $180-187$

20. Wang SY, Cowan JE, Cary KC, Chan JM, Carroll PR, Cooperberg MR (2014) Limited ability of existing nomograms to predict outcomes in men undergoing active surveillance for prostate cancer. BJU Int 114(6b):E18-E24

21. Moore CM, Ridout A, Emberton M (2013) The role of MRI in active surveillance of prostate cancer. Curr Opin Urol 23(3):261-267

22. Stamatakis L, Siddiqui MM, Nix JW et al (2013) Accuracy of multiparametric magnetic resonance imaging in confirming eligibility for active surveillance for men with prostate cancer. Cancer 119(18):3359-3366

23. Hoeks CM, Somford DM, van Oort IM et al (2014) Value of 3-T multiparametric magnetic resonance imaging and magnetic resonance-guided biopsy for early risk restratification in active surveillance of low-risk prostate cancer: a prospective multicenter cohort study. Invest Radiol 49(3):165-172

24. Eichler K, Hempel S, Wilby J, Myers L, Bachmann LM, Kleijnen J (2006) Diagnostic value of systematic biopsy methods in the investigation of prostate cancer: a systematic review. J Urol 175(5):1605-1612 
25. Chun FK, Briganti A, Graefen M et al (2007) Development and external validation of an extended 10-core biopsy nomogram. Eur Urol 52(2):436-444

26. Dong F, Kattan MW, Steyerberg EW et al (2008) Validation of pretreatment nomograms for predicting indolent prostate cancer: efficacy in contemporary urological practice. J Urol 180(1):150-154

27. Iremashvili V, Soloway MS, Pelaez L, Rosenberg DL, Manoharan M (2013) Comparative validation of nomograms predicting clinically insignificant prostate cancer. Urology. 81(6):1202-1208

28. Bul M, Delongchamps NB, Steyerberg EW et al (2011) Updating the prostate cancer risk indicator for contemporary biopsy schemes. Can J Urol. 18(2):5625-5629
29. Kweldam CF, Wildhagen MF, Bangma $\mathrm{CH}$, van Leenders GJ (2014) Disease-specific death and metastasis do not occur in patients with Gleason score $\leq 6$ on radical prostatectomy. BJU Int. doi: $10.1111 /$ bju. 12879

30. Van der Kwast T, Bubendorf L, Mazerolles C et al (2013) Guidelines on processing and reporting of prostate biopsies: the 2013 update of the pathology committee of the European Randomized Study of Screening for Prostate Cancer (ERSPC). Virchows Arch 463(3):367-377 